\title{
An image-based method to automatically propagate bony landmarks: application to computational spine biomechanics
}

\author{
Marcelo E. de Oliveira, Luiz M.G. Netto, Michael Kistler, Daniel \\ Brandenberger, Philippe Büchler \& Carol-C. Hasler
}

To cite this article: Marcelo E. de Oliveira, Luiz M.G. Netto, Michael Kistler, Daniel Brandenberger, Philippe Büchler \& Carol-C. Hasler (2015) An image-based method to automatically propagate bony landmarks: application to computational spine biomechanics, Computer Methods in Biomechanics and Biomedical Engineering, 18:14, 1535-1542, DOI: 10.1080/10255842.2014.927445

To link to this article: https://doi.org/10.1080/10255842.2014.927445

\section{曲 Published online: 24 Jun 2014.}

\section{Submit your article to this journal 주}

Шll Article views: 167

View Crossmark data ¿

Citing articles: 3 View citing articles $\square$ 


\title{
An image-based method to automatically propagate bony landmarks: application to computational spine biomechanics
}

\author{
Marcelo E. de Oliveira ${ }^{\mathrm{a}, \mathrm{b} *}$, Luiz M.G. Netto ${ }^{\mathrm{c}}$, Michael Kistler ${ }^{\mathrm{d}}$, Daniel Brandenberger ${ }^{\mathrm{e}}$, Philippe Büchler ${ }^{\mathrm{d}}$ and \\ Carol-C. Hasler ${ }^{\mathrm{e}}$ \\ ${ }^{a}$ Robotic Systems Laboratory, Swiss Federal Institute of Technology, Lausanne, Switzerland; ${ }^{b}$ Graduate School for Cellular and \\ Biomedical Sciences, University of Bern, Switzerland; ${ }^{c}$ Department of Material Sciences, State University of São Paulo, São Paulo, \\ Brazil; 'Institute for Surgical Technologie and Biomechanics, University of Bern, Bern, Switzerland; ${ }^{e}$ Orthopaedic Department, \\ University Children's Hospital, Basel, Switzerland
}

(Received 3 October 2012; accepted 20 May 2014)

\begin{abstract}
In attempts to elucidate the underlying mechanisms of spinal injuries and spinal deformities, several experimental and numerical studies have been conducted to understand the biomechanical behavior of the spine. However, numerical biomechanical studies suffer from uncertainties associated with hard- and soft-tissue anatomies. Currently, these parameters are identified manually on each mesh model prior to simulations. The determination of soft connective tissues on finite element meshes can be a tedious procedure, which limits the number of models used in the numerical studies to a few instances. In order to address these limitations, an image-based method for automatic morphing of soft connective tissues has been proposed. Results showed that the proposed method is capable to accurately determine the spatial locations of predetermined bony landmarks. The present method can be used to automatically generate patient-specific models, which may be helpful in designing studies involving a large number of instances and to understand the mechanical behavior of biomechanical structures across a given population.
\end{abstract}

Keywords: soft connective tissues; finite element meshes; image-based method; automatic morphing

\section{Introduction}

In order to understand the structural behavior of human spinal motion segments and to elucidate the underlying mechanisms of spine injuries and spinal dysfunctions, several in vivo and in vitro studies have been conducted in the last decades (Panjabi et al. 1976; Pearcy 1985; Wilke et al. 1995; Rozumalski et al. 2008). Based on these experimentally obtained results, physical and biomechanical properties were derived and incorporated into mathematical and computational models. Since its first clinical application in orthopedics by Brekelmans et al. (1972), the finite element method (FEM) has been widely used in biomechanics research, providing a better understanding of the behavior of biomechanical structures. This technique allowed the investigation of the influence of specific elements on the overall biomechanical system and at different levels of modeling abstraction, such as hardand soft-tissue anatomy, material behavior, mechanical loads, and boundary conditions.

The importance of using complex constitutive models for predicting stresses and strains in intervertebral discs was first demonstrated by the pioneer work of Klisch and Lotz (1999). In this work, a number of complex features including geometric and constitutive nonlinearities, anisotropy, and viscoelasticity were taken into account. The biomechanical behavior of the intervertebral disc was investigated under quasi-static loading conditions and subject to large deformations, providing important insights into the underlying mechanism of intervertebral disc injury. Since then, a number of clinically relevant problems using complex constitutive models have been proposed in spine biomechanics. In 1984, Yang and King (1984) proposed a facet-related hypothesis for explaining low back pain using FEM. In the 1990s, Bozic et al. (1994) and Yoganandan et al. (1996) studied the mechanical behavior of cervical vertebrae under axial compressive loads, showing the feasibility of using FEM to investigate the underlying mechanisms of spine injuries and its capability in predicting fracture patterns observed clinically. Mizrahi et al. (1993) have performed stress analysis of normal and osteoporotic lumbar vertebral bodies. However, these models are limited by unknown patient-specific mechanical properties, hardand soft-tissue geometries, and most of these models presented several convergence difficulties due to the complexity of the material laws, large displacements, and boundary conditions (Stokes et al. 1999) and, in addition, this modeling technique may be prohibitive for applications involving multiple spinal segments.

Recent works aiming at modeling the macroscopic behavior of spinal structures are based on the pioneer

*Corresponding author. Email: marcelo.eliasdeoliveira@epfl.ch 
experimental work of Panjabi et al. (1976), which developed an elegant experimental procedure and reported systematically obtained, three-dimensional flexibility and stiffness coefficients of spinal motion segments from healthy fresh human cadaver thoracic spines, considering coupling effects. Based on this work, Gardner-Morse et al. (1990) presented a method for deriving a spinal element stiffness matrix based on a shear beam model, matching numerical simulations to the reported experimental observations. Stiffness matrices representing the intrinsic mechanical properties of the spine were derived and have been used by several authors to address spine-related problems and optimization of orthopedic implants (Vanderby et al. 1986; Ghista et al. 1988; Gardner-Morse and Stokes 1994; Dumas et al. 2005; Elias de Oliveira, Hasler et al. 2011).

Most of the currently used biomechanical models are constructed by assuming material properties derived from the previous experimental works and for this reason, some quantitative disagreements between numerical and experimental studies reported in the earlier investigations may be attributed mainly to geometrical aspects and boundary conditions (Panjabi et al. 1994; Camacho et al. 1997; Nightingale et al. 2002; Wheeldon et al. 2006; Del Palomar et al. 2008). These disagreements may possibly be explained by the fact that in mesh-based models, hardand soft-tissue geometries details, fibers orientation, ligament attachment points, and nucleus pulposus position, as well as boundary conditions, are user-defined parameters.

In 2007, Noailly et al. (2007) has proposed a systematic methodology to generate a class of equivalent geometric models from a single lumbar spine bi-segment finite element model subjected to axial, sagittal, and frontal pure rotational moments. In this study, it has been found that the stress distributions and strain energies in different spine structures are sensitivity to minor geometrical changes, and that the predicted overall ranges of motion are invariant when considering equivalent geometric models.
In order to improve the modeling reliability in meshbased models, an automated procedure capable of propagating predetermined soft connective tissues structures, boundary conditions, and load locations has been proposed in this work. The proposed method is based on image-registration techniques, enabling large populationbased studies as well as accelerating the process of patientspecific modeling. Such a technique is also expected to reduce the intra- and interobserver modeling variability.

\section{Methods}

\subsection{Data sets}

Magnetic resonance imaging data sets (16 bits, with voxel size $1.0 \times 1.0 \times 1.1 \mathrm{~mm}^{3}$ ) were acquired from five individuals (mean age $14 \pm 1.5$ years) suffering from adolescent idiopathic scoliosis (AIS) with moderate to severe thoracic deformities. For each data set, the apex of the primary curve was identified, and the vertebral bodies locate at the apex, one level below and one level above the apex were segmented by an expert in spinal disorders. Thus, a total of 15 thoracic vertebrae ranging from Th5 to Th12 were segmented.

\subsection{Intra- and interobserver landmark positioning variability}

In order to assess the intra- and interobserver variability, a custom-made software has been developed using the GNU $\mathrm{C}++$ compiler $(\mathrm{g}++4.6 .1)$ and the Visualization Toolkit library (VTK 5.6, http://www.vtk.org). This software allows the visualization of vertebral bone surface meshes, providing the user with different interaction mechanisms for mouse, key, and callback events for interactive visualization, selection of predetermined vertebral bone landmarks, and data storage for post-processing analysis. All volunteers with experience on spinal biomechanics were trained on how to use the software and instructed to place the vertebral bone landmarks at predetermined locations and in a predetermined sequence (Figure 1). (a)

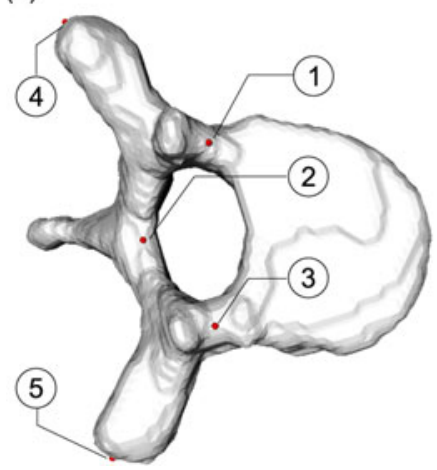

(b)

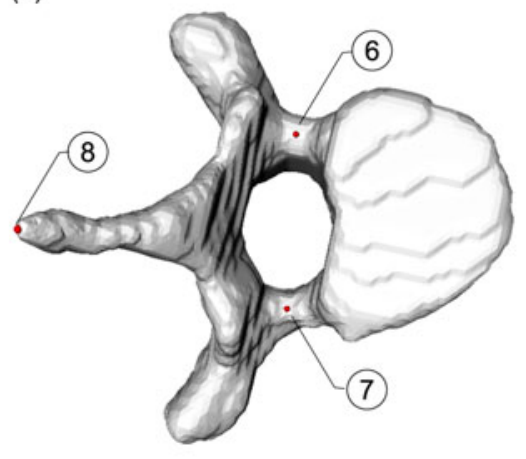

(c)

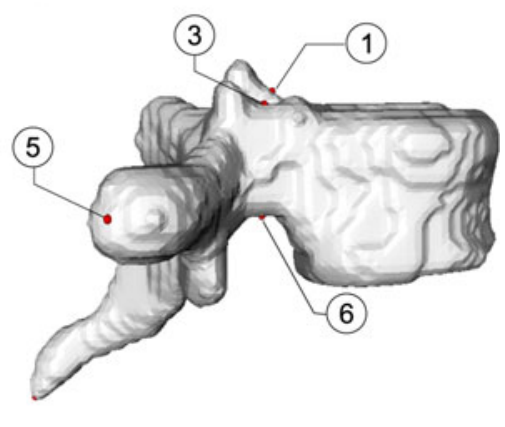

Figure 1. Vertebral anatomical landmarks used to assess the intra- and interobserver reliability and to validate the proposed image-based registration technique. 
The precise localization of anatomical landmarks relies on the level of anatomical details of the organ of interest and is observer dependent. In this sense, the variability of a specific anatomical landmark by one observer on one specific data set, and the variance between different examiners on different data sets may exist, and, therefore, should be investigated. In order to assess the intra- and interobserver landmark positioning variability in biomechanics, six volunteers working in spine biomechanics have been enrolled in this study. A randomly selected volunteer has been asked to perform the identification of eight predefined vertebral bone landmarks, and interspinous and intertransverse ligaments attachment points in the template surface mesh, as shown in Figures 1 and 4, respectively. Superior and inferior vertebral endplates have been automatically detected based on the technique described elsewhere (Elias de Oliveira, Neto et al. 2011)]. The five other volunteers were included in the intra-and interobserver reliability analyses using the statistical approach proposed by Eliasziw et al. (1994), as described next. Five volunteers $(o=5)$ working in the field of spinal biomechanics were recruited and asked to determine three repeated times $(m=3)$ the 3D spatial locations of eight different anatomical landmarks on three randomly selected thoracic vertebrae iso-surfaces $(n=3 \times 8=24)$. A total of $360(m \times n \times o)$ distinct positioning of landmarks were performed, and the participants were not consciously aware of the temporal data sequence or that the same vertebra would be considered more than once. The $k$ th $(k=1, \ldots, m)$ landmark positioning performed by the $j$ th $(j=1, \ldots, o)$ observer on the $i$ th $(i=1, \ldots, n)$ anatomical landmark is denoted by $Y_{i j k}$, where the $Y_{i j k}$ is the Euclidean distance between the individually selected landmarks and its respective calculated centroid considering all the 15 selected landmarks per anatomical location $(o \times m)$, as shown in (Figure 2). Thus, the spatial positioning of all different anatomical landmarks can be

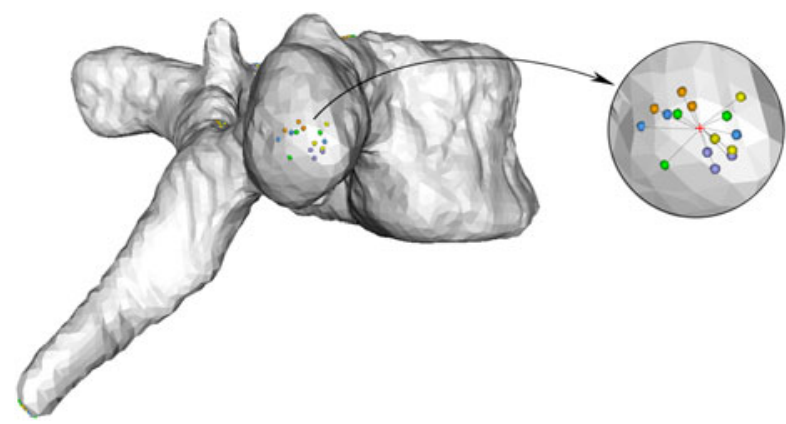

Figure 2. The intra- and interobserver landmark positioning variability representation. It has been assessed by considering the $L_{2}$-norm components in the $x, y$, and $z$ directions between the individually selected landmarks and its respective calculated centroid (arithmetic mean of all manually selected landmarks), represented by a red cross symbol.

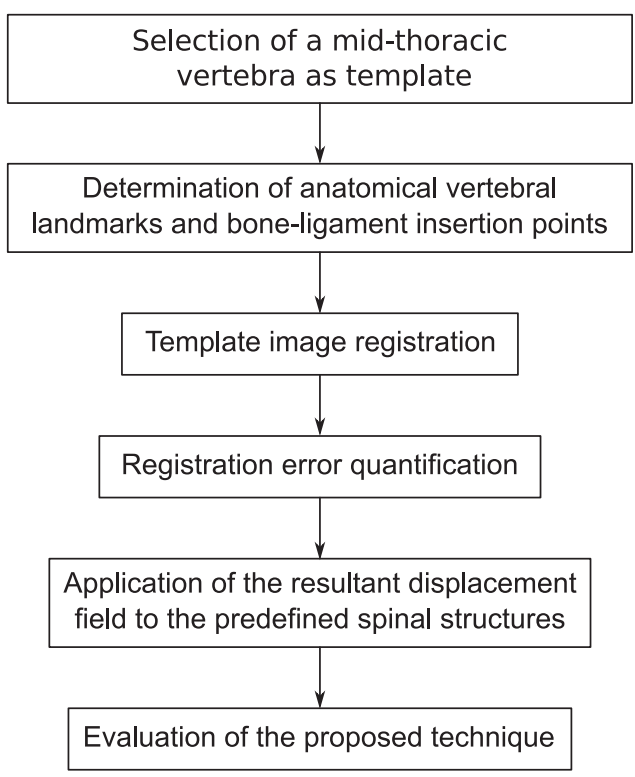

Figure 3. Flowchart summarizing the methodology proposed for consistent mesh-based modeling.
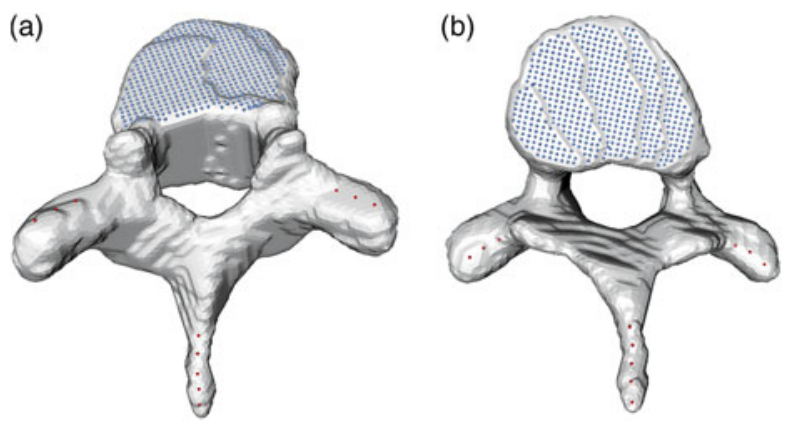

Figure 4. Skeletal connective tissues attachment points and the automatically detected superior and inferior vertebral endplates are represented in red and blue in the template surface mesh, respectively.

represented according to the following model:

$$
Y_{i j k}=\mu+S_{i}+O_{j}+(S O)_{i j}+e_{i j k}
$$

where $\mu$ is the grand mean computed base on all threedimensional spatial locations; $S_{i}$ the effect of data set $i$ (i.e., difference between $\mu$ and the mean value for data set i); $O_{j}$ is the difference between $\mu$ and the mean value of observations by observer $j$; $(S O)_{i j}$ is the heterogeneity term; and $e_{i j k}$ is the error in measurements by a single observer on a single data set. We assume that $S_{i}, O_{j},(S O)_{i j}$, and $e_{i j k}$ are independent normal variables with variances $s_{\mathrm{b}}^{2}, s_{\mathrm{o}}^{2}, s_{\mathrm{h}}^{2}$, and $s_{\mathrm{w}}^{2}$, respectively, where $s_{\mathrm{b}}$ is the variance between data sets; $s_{\mathrm{O}}$ is the variance between observers; $s_{\mathrm{h}}$ is the variance between different observers on different 
(a)

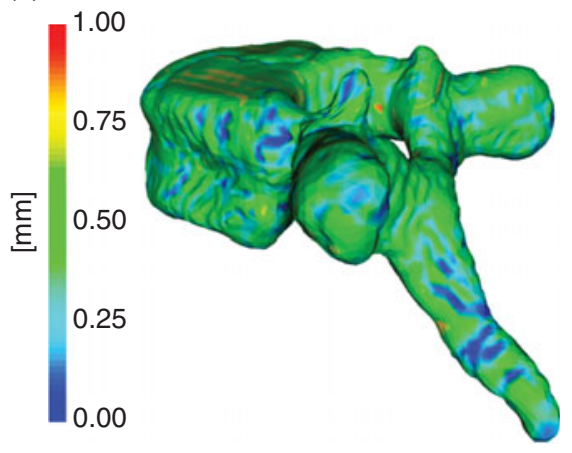

(b)

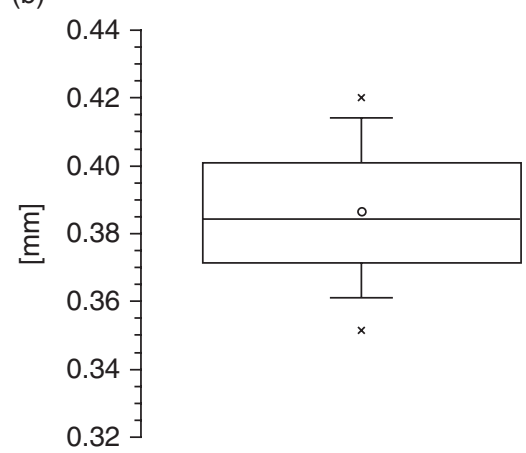

Figure 5. (a) Minimum distance map representing the dissimilarity between the float and the reference image iso-surfaces (mm). (b) Box-plot showing the median, upper and lower quartiles, and outliers of the mean minimum $L_{2}$-norms computed for all 14 registered thoracic vertebrae.

data sets; and $s_{\mathrm{w}}$ is the variance of observations by one observer on one data set. Therefore, the variance is given by the sum of all independent components:

$$
s^{2}=s_{\mathrm{b}}^{2}+s_{\mathrm{o}}^{2}+s_{\mathrm{h}}^{2}+s_{\mathrm{w}}^{2}
$$

and the intra- and inter-class correlation coefficients are defined, respectively, as follows:

$$
\begin{gathered}
\rho_{\text {intra }}=\frac{s_{\mathrm{b}}^{2}}{\left(s_{\mathrm{b}}^{2}+s_{\mathrm{w}}^{2}\right)}, \\
\rho_{\text {inter }}=\frac{s_{b}^{2}}{\left(s_{b}^{2}+s_{o}^{2}+s_{h}^{2}+s_{w}^{2}\right)}
\end{gathered}
$$

\subsection{Template matching approach and dissimilarity measure}

The predefined supra interspinous and intertransverse ligaments were used as anatomical descriptors, and the preselected mid thoracic vertebra image was registered to all remaining data sets using rigid, affine, and non-rigid techniques in a subject-specific fashion (Ourselin et al. 2000; Mattes 2001; Vercauteren et al. 2007). The registration accuracy was quantitatively assessed using the mean minimum $L_{2}$-norm, and the resulting displacement fields were applied to the surface of the template mesh containing the predetermined spinal structures. This process provided an automatic morphing of the identified spinal structures to all registered instances. The proposed methodology is summarized in the flowchart Figure 3.

\section{Results}

The accuracy of the registration was quantitatively assessed based on the mean minimum $L_{2}$-norms (Figure 5 (a)). In this context, this measure quantifies the dissimilarity between the reference image and the target image transformed using the displacement field computed in the image-registration procedure. The comparisons have been repeated for each of the 14 vertebrae of the database. The results showed that the mean registration error was below $0.39 \mathrm{~mm}$ and the maximal error remained lower than $0.43 \mathrm{~mm}$ (Figure 5(b)). This level of registration accuracy indicates that the calculated displacement fields can be used to provide an accurate estimate of the spatial location of the spinal structures present on the surface of the template mesh (Figures 6 and 8).

Intra- and interobserver variability in the landmark positioning was assessed based on the recorded manual landmark selection. An average distance of less than $1 \mathrm{~mm}$ has been observed between the individual landmark positions and the centroid of all manually selected landmarks (Figures 7(a)-(c)).

The analysis of variance for determining intra- and interobserver observer reliability based on repeated positioning of landmarks measurements is shown in Table 1. Intraclass and interclass correlations coefficients were computed according to the technique summarized in Section 2.2. Intraobserver Euclidean estimates have 0.37 variability (within-subject standard deviation) and $\rho_{\text {intra }}=37.45 \%$

Table 1. Analysis of variance table for determining intra- and interobserver reliability based on repeated positioning of landmarks.

\begin{tabular}{lccc}
\hline Source of variation & $\begin{array}{c}\text { Degrees of } \\
\text { freedom }\end{array}$ & $\begin{array}{c}\text { Sum of } \\
\text { squares }\end{array}$ & $\begin{array}{c}\text { Observed } \\
\text { mean square }\end{array}$ \\
\hline Data sets & 23 & 32.342 & 1.4062 \\
Observer & 4 & 1.0170 & 0.25424 \\
Data sets $\times$ Obs & 92 & 18.095 & 0.19669 \\
Residual & 240 & 32.321 & 0.13467 \\
Total & 359 & 83.776 & \\
\hline
\end{tabular}

Note: Each anatomical landmark has been selected three times by each observer. 
(a)

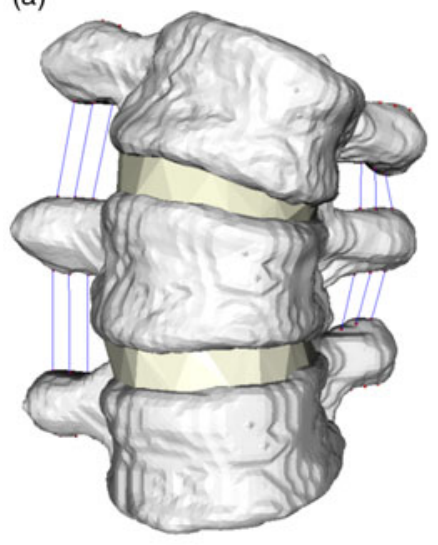

(c)

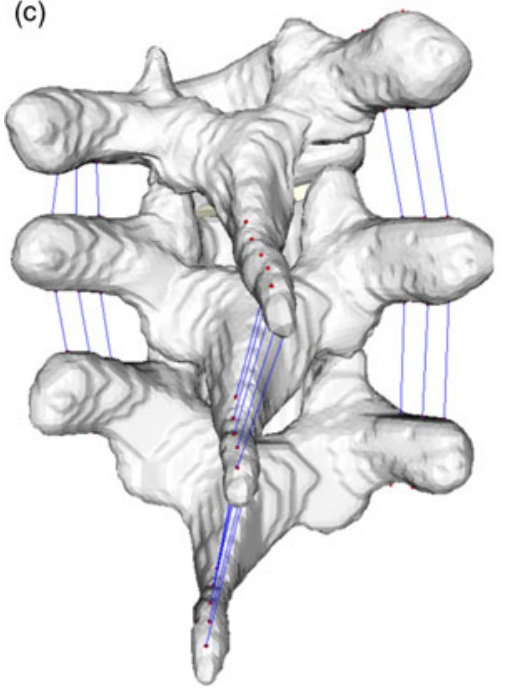

(b)

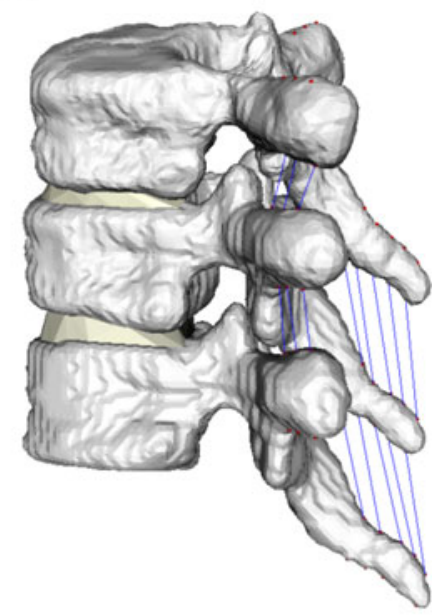

(d)

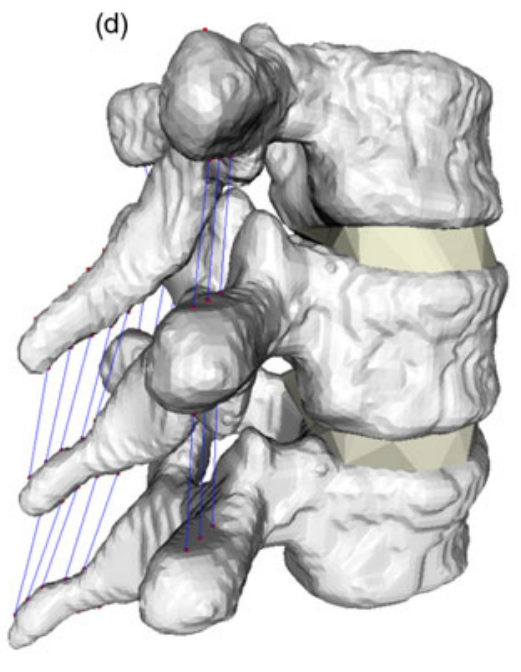

Figure 6. (a)-(d) Anterior, lateral, and posterior views of two adjacent spinal segments with their respective reconstructed intervertebral discs, and associated supra interspinous and intertransverse ligaments. The predicted ligament attachment points are highlighted in red.

reliability, and interobserver estimates have 0.38 variability (within-subject standard deviation) and $\rho_{\text {inter }}=35.33 \%$ reliability, thus, showing that the spatial locations of spinal anatomical landmarks cannot be consistently reproduced using manual landmark-based methods due to its low reliability (Figures 7 (a)-(c)).

The displacement fields obtained from the registration procedure were used to automatically determine the spatial location of the predetermined spinal structures (vertebral landmarks, ligament attachment points, and vertebral endplates), as shown in Figures 6 and 8. These automatically determined positions were compared with the average position obtained using manual landmark identification. The root-mean-square error considering all 24 spinal landmarks (three vertebrae $\times$ eight landmarks) was $0.60 \pm 0.44 \mathrm{~mm}$. This result indicates that the imageregistration method is able to predict accurately the spatial location of predetermined anatomical landmarks (Figure 8).
Thus, showing that the image-registration morphing method was able to predict the spatial location of the eight predetermined anatomical landmarks, proving to be suitable for predicting more complex spinal anatomical structures such as ligament attachment points and vertebral endplates.

\section{Discussion}

The traditional manual-based approach commonly used in biomechanics for determining structures of interest requires many iterations, which makes this process time consuming and error-prone, especially when a large number of soft connective tissues, loads, and boundary conditions are taken into account. With the development of patient-specific modeling, the need for an automatic tool to generate FE models is increasing. In this study, an imaged-based registration method has been presented to 

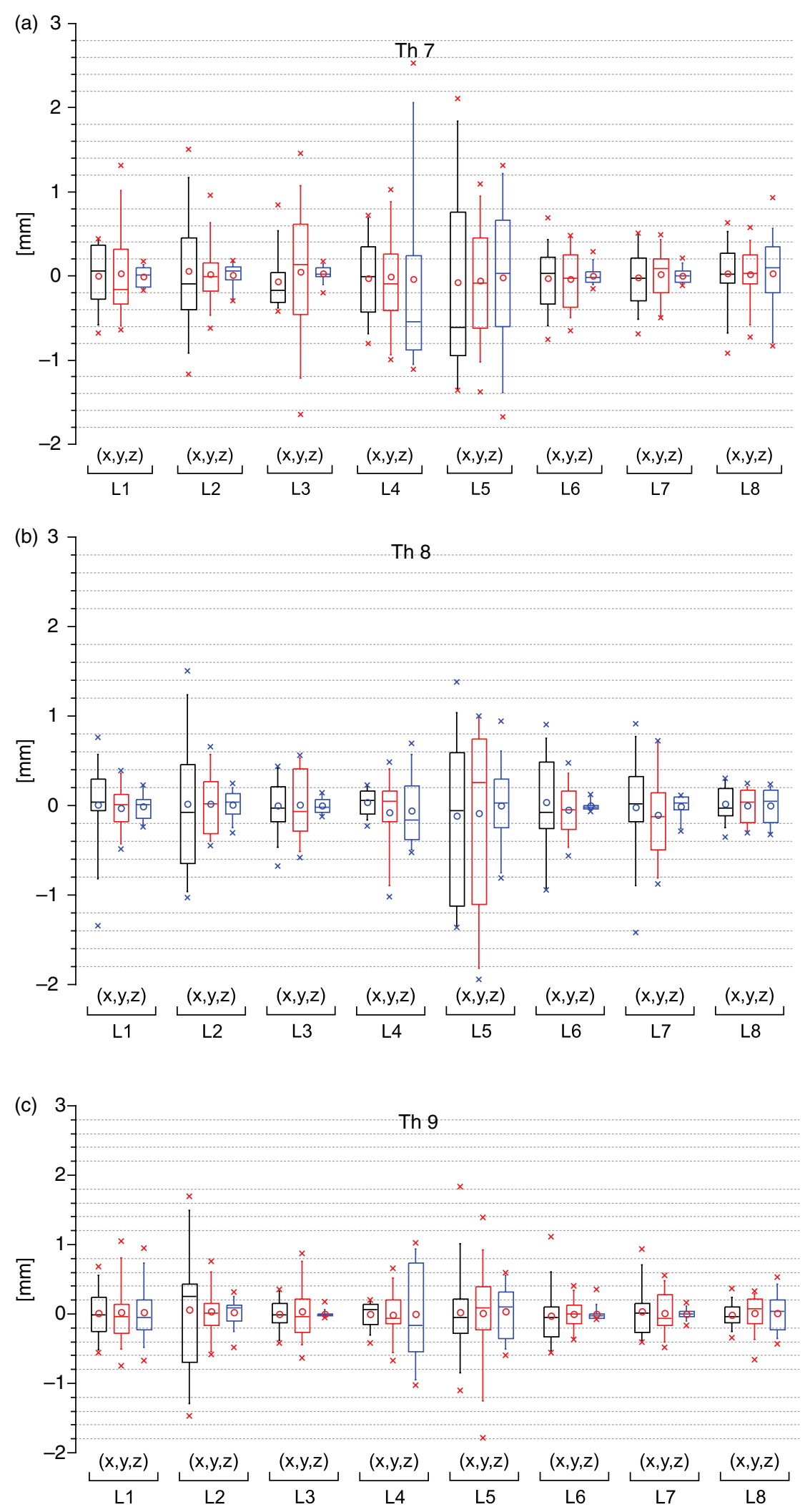

Figure 7. Box plots showing the mean centered bone landmarks spatial locations distributions with respect to $x, y$, and $z$ axes of the thoracic vertebrae Th7, Th8, and Th9 shown in Figure $8(n=15$, every landmark has been identified three repeated times by five different volunteers). 


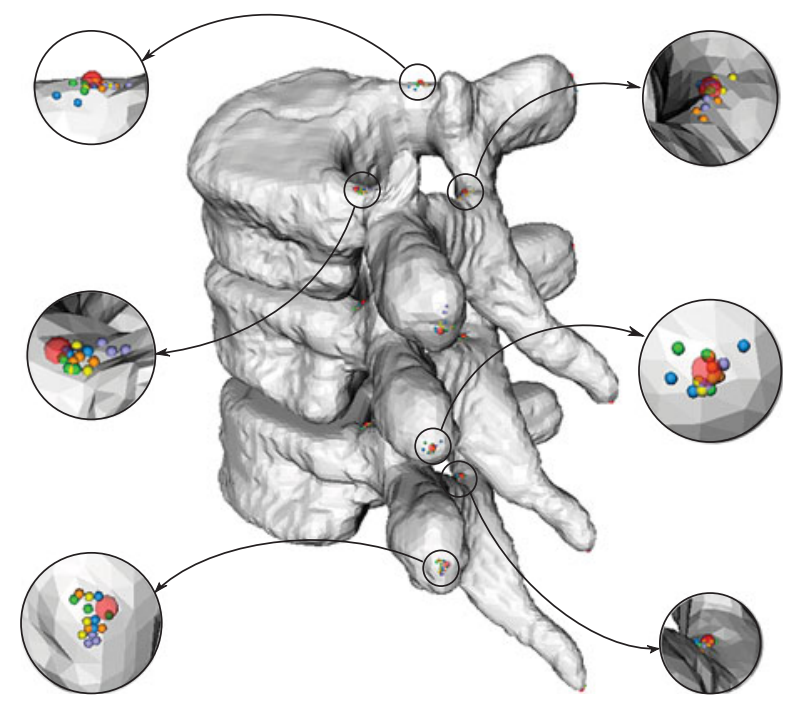

Figure 8. Automated morphing of vertebral landmarks based on the image registration-based method against the manual selection approach. Thoracic levels Th7, Th8, and Th9, from the top to the bottom, respectively.

automatically propagate anatomical structures identified on a template reference mesh to any new structure with similar characteristics.

In addition, a low intra- and interobserver reliability has been verified in the manual-based method, which indicates a limited reproducibility of models generated using this approach. The reliability of this method is sensitive to the anatomy of the structures of interest, thus indicating that the reliability level is associated with the geometrical complexity of the organ of interest. It is important to note that the eight predefined vertebral landmarks used in this study are considerably easier to be identified than those used as ligament attachment points, vertebral endplates or boundary conditions. For this reason, an even lower reliability can be expected for structures without strict boundaries, such as the supra interspinous and intertransverse ligaments and, therefore, such structures were not used in the reliability analysis. These observations are in accordance with our results, showing that the individual variance depends on the specific landmark position as well as on the shape of the bone surface; for example, landmark L4 exhibits a large variance in the manual selection for the thoracic vertebra Th7, while the same landmark has been consistently identified on vertebrae Th8 and Th9. Overall, a higher location variability was observed for landmarks L2, L4, and L5 (Figures 7(a)-(c)).

The motivation for considering data sets acquired from patients suffering from AIS is given by the fact that this pathology is often accompanied or followed by vertebral bonny changes and, consequently, asymmetries are often induced as well. Furthermore, this neuromuscular disorder induces three-dimensional rotations and translations of individual vertebrae along the spinal column. For these reasons, the identification of ligaments attachment points, vertebral endplates, and boundary conditions becomes even more challenging than in most of the traditional applications in biomechanics.

The method presented in this study can be easily applied to any other biomechanical problem, where the geometry and boundary conditions play an important role. The proposed morphing technique allows the standardization of subject-specific models. A database containing anatomical structures of interest could be developed by anatomists and, thus, these templates could then be used to automatically morph these predefined structures of interest to any new organ having similar morphological properties, ensuring consistent modeling across research teams.

The accurate prediction of anatomical structures of interest is dependent of the registration accuracy; thus the proposed morphing technique is recommended only when a good surface-to-surface mapping can be established. For this reason, although the method is automatic, the quality of the surface registration must be carefully evaluated before applying the resulting displacement fields to the anatomical structures of interest. However, the measurement of registration accuracy can be easily performed by the simple calculation of the Hausdorff and/or of the mean minimum $L_{2}$-norms, which do not require necessarily oneto-one correspondences.

The proposed method for automatic morphing encodes anatomical structures of the region of interest, as the correspondence between similar anatomical structures is inherited from the image-registration procedure, thus suggesting that this technique enables an automatic and consistent generation of patient-specific mesh-based models.

\section{References}

Bozic KJ, Keyak JH, Skinner HB, Bueff HU, Bradford DS. 1994. Three-dimensional finite element modeling of a cervical vertebra: an investigation of burst fracture mechanism. J Spinal Disord. 7(2):102-110.

Brekelmans WA, Poort HW, Slooff TJ. 1972. A new method to analyse the mechanical behaviour of skeletal parts. Acta Orthop Scand. 43(5):301-317.

Camacho DL, Nightingale RW, Robinette JJ, Vanguri SK, Coates DJ, Myers BS. 1997. Experimental flexibility measurements for the development of a computational head-neck model validated for near-vertex head impact., p. 473-486 SAE Technical Paper 973345.

Del Palomar AP, Calvo B, Doblaré M. 2008. An accurate finite element model of the cervical spine under quasi-static loading. J Biomech. 41(3):523-531.

Dumas R, Lafage V, Lafon Y, Steib JP, Mitton D, Skalli W. 2005. Finite element simulation of spinal deformities correction by in situ contouring technique. Comput Methods Biomech Biomed Eng. 8(5):331-337.

Elias de Oliveira M, Hasler CC, Zheng G, Studer D, Schneider J, Büchler P. 2011. A multi-criteria decision support for 
optimal instrumentation in scoliosis spine surgery. Struct Multidisc Optim. 45:917-929.

Elias de Oliveira M, Neto LMG, May C, Büchler P. 2011. Automatic detection of vertebral endplates. In: XXIII congress of the international society of biomechanics, 2011; Brussels, Belgium.

Eliasziw M, Young SL, Woodbury MG, Fryday-Field K. 1994. Statistical methodology for the concurrent assessment of interrater and intrarater reliability: using goniometric measurements as an example. Phys Ther. 74(8):777-788.

Gardner-Morse M, Stokes IA. 1994. Three-dimensional simulations of the scoliosis derotation maneuver with CotrelDubousset instrumentation. J Biomech. 27(2):177-181.

Gardner-Morse MG, Laible JP, Stokes IA. 1990. Incorporation of spinal flexibility measurements into finite element analysis. J Biomech Eng. 112(4):481-483.

Ghista DN, Viviani GR, Subbaraj K, Lozada PJ, Srinivasan TM, Barnes G. 1988. Biomechanical basis of optimal scoliosis surgical correction. J Biomech. 21(2):77-88.

Klisch SM, Lotz JC. 1999. Application of a fiber-reinforced continuum theory to multiple deformations of the annulus fibrosus. J Biomech. 32(10):1027-1036.

Mattes D. 2001. Nonrigid multimodality image registration. In: Sonka M, Hanson KM, editors. Proceedings of SPIE. Society of photo-optical instrumentation engineers (SPIE) conference series, 2001; vol. 4322. SPIE. p. 1609-1620.

Mizrahi J, Silva MJ, Keaveny TM, Edwards WT, Hayes WC. 1993. Finite-element stress analysis of the normal and osteoporotic lumbar vertebral body. Spine. 18 (14):2088-2096.

Nightingale RW, Winkelstein BA, Knaub KE, Richardson WJ, Luck JF, Myers BS. 2002. Comparative strengths and structural properties of the upper and lower cervical spine in flexion and extension. J Biomech. 35(6):725-732.

Noailly J, Wilke HJ, Planell Ja, Lacroix D. 2007. How does the geometry affect the internal biomechanics of a lumbar spine bi-segment finite element model? Consequences on the validation process. J Biomech. 40(11):2414-2425.

Ourselin S, Roche A, Prima S, Ayache N. 2000. Block matching: a general framework to improve robustness of rigid registration of medical images. In: Delp S, DiGioia AM, Jaramaz B, editors. Medical image computing and computer assisted intervention. MICCAI 2000, vol. 1935. NewYork: Springer; p. 557-566.

Panjabi MM, Brand RA, White AA. 1976. Three-dimensional flexibility and stiffness properties of the human thoracic spine. J Biomech. 9(4):185-192.

Panjabi MM, Lydon C, Vasavada A, Grob D, Crisco JJ, Dvorak J. 1994. On the understanding of clinical instability. Spine. 19(23):2642-2650.

Pearcy MJ. 1985. Stereo radiography of lumbar spine motion. Acta Orthop Scand. 212(21):1-45.

Rozumalski A, Schwartz MH, Wervey R, Swanson A, Dykes DC, Novacheck T. 2008. The in vivo three-dimensional motion of the human lumbar spine during gait. Gait Posture. 28 (3):378-384

Stokes I, Gardner-Morse M, Aubin C, Labelle H. 1999. Biomechanical simulations for planning of scoliosis surgery. Stud Health Technol Inform. 59:343-346.

Vanderby R, Daniele M, Patwardhan A, Bunch W. 1986. A method for the identification of in-vivo segmental stiffness properties of the spine. J Biomech Eng. 108(4):312-316.

Vercauteren T, Pennec X, Perchant A, Ayache N. 2007. Nonparametric diffeomorphic image registration with the demons algorithm. Med Image Comput Comput Assist Interv. 10(Pt 2):319-326.

Wheeldon JA, Pintar FA, Knowles S, Yoganandan N. 2006. Experimental flexion/extension data corridors for validation of finite element models of the young, normal cervical spine. J Biomech. 39(2):375-380.

Wilke HJ, Wolf S, Claes LE, Arand M, Wiesend A. 1995. Stability increase of the lumbar spine with different muscle groups: a biomechanical in vitro study. Spine. 20 (2):2168-2169.

Yang KH, King AI. 1984. Mechanism of facet load transmission as a hypothesis for low-back pain. Spine. 9(6):557-565.

Yoganandan N, Kumaresan S, Voo L, Pintar FA. 1996. Finite element applications in human cervical spine modeling. Spine. 21(15):1824-1834. 